\title{
Dinâmica de Transposição de herbicida Através de Palha de Aveia-Preta Utilizando Diferentes Pontas de Pulverização ${ }^{1}$
}

\author{
Dynamics of Herbicide Crossing Through Black Oat Straw by Using Different Nozzle Models
}

\author{
COSTA, A.G.F. ${ }^{2}$, MACIEL, C.D.G. ${ }^{2}$, VELINI, E.D. ${ }^{3}$ e IKEDA, F.S. ${ }^{4}$
}

\begin{abstract}
RESUMO - A transposição da palha por herbicidas aplicados em pré ou pós-emergência durante a aplicação é determinante na sua eficiência, dinâmica e impacto ambiental. $O$ experimento foi conduzido no Núcleo de Pesquisas Avançadas em Matologia - FCA/UNESP, campus de Botucatu-SP, tendo como objetivo avaliar o desempenho de diferentes modelos de pontas de pulverização na transposição em quantidades crescentes de palha de aveiapreta (Avena strigosa). Os tratamentos foram constituídos pelo monitoramento do traçador corante Azul Brilhante (FDC-1) a 3.000 ppm, pulverizado com as pontas de pulverização XR11002-VS, TJ60-11002VS, FL-5VS, DG11002-VS, TXVK-8, TT11002-VP e AI11002-VS, utilizando, respectivamente, as pressões de trabalho de 1,$4 ; 2,0 ; 1,5 ; 2,0 ; 4,9 ; 3,0$ e 3,0 $\mathrm{kgf} \mathrm{cm}^{-2}$ e volume de calda de 200, 200, 428, 200, 213 e $270 \mathrm{~L} \mathrm{ha}^{-1}$ sobre quantidades de 0, 1, 2, 4, 6, 8,10 e $12 \mathrm{t} \mathrm{ha}^{-1}$ de palha de aveia-preta. O delineamento utilizado foi o inteiramente casualizado, com sete tratamentos e cinco repetições, as quais foram constituídas de caixas plásticas com palha acondicionada sobre um fundo falso de área conhecida, sendo este lavado após as aplicações, para posterior quantificação do traçador em espectrofotometria. $\mathrm{O}$ modelo de Mitscherlich simplificado $\left(\mathrm{Y}=10^{\wedge}\left(2-\left(\mathrm{C}^{*} \mathrm{X}\right)\right)\right)$ mostrou ajuste satisfatório para os dados originais de traçador que transpôs a palha, apresentando coeficientes de determinação $\left(R^{2}\right)$ elevados, oscilando entre 0,9782 e 0,9971. Todos os modelos de pontas de pulverização mostraram-se similares na transposição da palha pelo traçador. As porcentagens médias de transposição foram de 43,00;18,77;3,73;0,78;0,17;0,04 e 0,01\% para as quantidades de $1,2,4,6,8,10$ e $12 \mathrm{t} \mathrm{ha}^{-1}$ de palha, respectivamente.
\end{abstract}

Palavras-chave: ponta de pulverização, Avena strigosa, cobertura morta, corante FDC-1.

\begin{abstract}
Straw crossing by pre- or post-emergence herbicide application determines its effectiveness, dynamics and environmental impact. The trial was carried out at NuPAM - FCA/ UNESP, Botucatu Campus, São Paulo, Brazil, to evaluate the performance of different nozzle models in crossing increasing amounts of black oat (Avena strigosa) straw. The treatments consisted of monitoring Brilliant Blue (FDC-1) tracer dye at 3.000 ppm, sprayed with nozzles XR11002-VS; TJ60-11002VS; FL-5VS; DG11002-VS; TXVK-8; TT11002-VP and AI11002-VS, at work pressures 19.9; 28.4; 21.3; 28.4; 69.7; 42.7 and 42.7 PSI, respectively; and mix volumes 200;

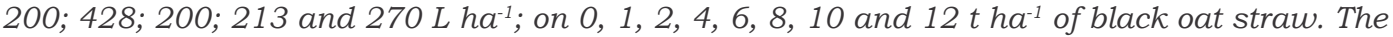
experimental design was entirely randomized with seven treatments and five replications, constituted by plastic boxes packed with straw over a fake bottom of known area, washed after the applications, for posterior tracer quantification by spectrophotometry. The Simplified Mitscherlich model $\left(Y=10^{\wedge}\left(2-\left(C^{*} X\right)\right)\right)$ showed a satisfactory adjustment for the tracer original data crossing the straw, presenting high determination coefficients $\left(R^{2}\right)$ between 0.9782 and 0.9971 . The nozzle models were similar in relation to straw crossing by the tracer. The mean crossing percentages were 43.00; 18.77; 3.73; 0.78; 0.17; 0.04 and 0.01\% for the amounts $1,2,4,6,8,10$ and $12 t^{\text {tha }}{ }^{-1}$ of black oat straw, respectively.
\end{abstract}

Key words: nozzle, Avena strigosa, mulch, FDC-1 tracer.

Recebido para publicação em 19.5.2004 e na forma revisada em 17.12.2004.

Eng.-Agrônomo, M.S., doutorando em Agricultura, FCA/UNESP. Caixa Postal 237, 18603-970 Botucatu-SP. ${ }^{3}$ Prof. Assistente, D.S., Dep. de Produção Vegetal - FCA/UNESP. ${ }^{4}$ Eng.-Agrônoma, FCA/UNESP. 


\section{INTRODUÇÃO}

Nos últimos anos, tem-se observado no Brasil uma rápida expansão da área total cultivada em que é utilizado algum tipo de cobertura morta. Entre os diferentes sistemas de cultivo, o plantio direto com culturas anuais apresenta crescente nível de adoção (Velini \& Negrisoli, 2000). Dessa forma, a interação de herbicidas aplicados em pré-emergência com os resíduos culturais é uma condição importante na conservação das práticas de plantio direto, uma vez que $60 \%$ ou mais da superficie do solo pode estar coberta, proporcionando interceptação da difusão de parte dos herbicidas aplicados (Marin et al., 1978).

Muitos agricultores aumentam as doses dos herbicidas residuais em plantio direto, alegando que parte do produto fica retida na palha e não atinge o solo. Nesse caso, evidentemente, o desperdício de produto onera o custo de produção e polui o ambiente (Rodrigues et al., 1998). Em alguns casos, aumenta-se também a pressão e a vazão do pulverizador, visando, inutilmente, facilitar a penetração do produto na camada de palha (Fornarolli, 1997).

Rodrigues (1993) destaca, entre outros fatores, que a quantidade e origem da cobertura morta podem influenciar a capacidade de um herbicida residual atingir o solo, no sistema de plantio direto. Almeida (1988), utilizando diversas coberturas mortas, verificou que os resíduos de aveia são, depois do nabo-forrageiro, os que se decompõem mais rapidamente; contudo, em função da maior massa inicial produzida, eles mantêm, por mais tempo, maior quantidade de cobertura morta sobre o terreno.

Vários estudos têm indicado a retenção de herbicidas aplicados em pré-emergência sobre a cobertura morta de aveia (Pastana, 1972; Rodrigues, 1993; Fornarolli et al., 1998; Rodrigues et al., 1998, 1999, 2000; Maciel, 2001; Cavenaghi et al., 2002a, b; Tofoli et al., 2002a, b). Em alguns casos, essa retenção pode ser um problema ainda maior se o herbicida não for lixiviado para o solo com as chuvas posteriores, em razão das características do herbicida e do ambiente. A maioria desses estudos utilizou em sua metodologia pontas de pulverização convencionais de jato plano tipo leque.
$\mathrm{O}$ que se chama genericamente de bico de pulverização é o conjunto de peças colocadas no final do circuito hidráulico do pulverizador, através do qual a calda é emitida para fora da máquina. Esse conjunto é composto de várias partes, das quais a ponta de pulverização é a mais importante, sendo responsável pela vazão, pelo tamanho das gotas e pela forma do jato emitido (Christofoletti, 1999a). De acordo com a Fundação ABC (1996), a seleção e operação das pontas de pulverização são passos importantes para aplicações precisas, as quais proporcionam reduções de perdas de produto, prejuízos e contaminação ambiental.

Segundo Christofoletti (1999b), a escolha de uma ponta de pulverização para aplicação de herbicidas em área conduzida sob sistema de plantio direto está relacionada com o tipo de pulverização que se pretende produzir, não existindo uma ponta universal capaz de atender todas as necessidades do agricultor.

Em razão da falta de estudos sobre o potencial de pontas de pulverização para herbicidas aplicados em pré-emergência no sistema de plantio direto, este ensaio objetivou avaliar o desempenho de diferentes modelos de pontas de pulverização na transposição de quantidades crescentes de palha de aveia-preta (Avena strigosa).

\section{MATERIAL E MÉTODOS}

O experimento foi conduzido em laboratório no Núcleo de Pesquisas Avançadas em Matologia (NuPAM), pertencente ao Departamento de Produção Vegetal da Faculdade de Ciências Agronômicas - UNESP, campus de Botucatu-SP.

O delineamento experimental utilizado foi o inteiramente casualizado, com sete tratamentos e cinco repetições. Os tratamentos estudados foram representados pela deposição da pulverização desenvolvida pelos modelos de pontas: XR11002-VS, TJ60-11002VS, FL-5VS, DG11002-VS, TXVK-8, TT11002-VP e AI11002-VS, respectivamente utilizadas em pressão de trabalho de $1,4{\mathrm{kgf} \mathrm{cm}^{-2}}^{-2}$ (200 L ha-1); 2,0 kgf cm${ }^{-2}$ (200 L ha-1); 1,5 $\mathrm{kgf} \mathrm{cm}^{-2}$ $\left(428 \mathrm{~L} \mathrm{ha}^{-1}\right) ; 2,0 \mathrm{kgf} \mathrm{cm}{ }^{-2}\left(200 \mathrm{~L} \mathrm{ha}^{-1}\right)$; $4,9 \mathrm{kgf} \mathrm{cm}^{-2} \quad\left(213 \mathrm{~L} \mathrm{ha}^{-1}\right) ; 3,0 \mathrm{kgf} \mathrm{cm}^{-2}$ (270 $\left.\mathrm{L} \mathrm{ha}^{-1}\right)$; e $3,0 \mathrm{kgf} \mathrm{cm}^{-2}\left(250 \mathrm{~L} \mathrm{ha}^{-1}\right)$. 
Em todos os tratamentos avaliou-se a quantidade de deposição da pulverização que conseguiu transpor as quantidades de $0,1,2$, 4, 6, 8, 10 e $12 \mathrm{t} \mathrm{ha}^{-1}$ de palha de aveia-preta (Avena strigosa), distribuídas na superficie do solo. A palha de aveia-preta foi coletada em área de produção de sementes, sendo os fragmentos da cobertura morta coletados após a colheita mecanizada dos grãos; posteriormente ela foi submetida à secagem em estufa de circulação de ar a $60^{\circ} \mathrm{C}$, durante três dias antes da instalação do experimento.

As unidades experimentais foram representadas por recipientes plásticos (caixas) com área de superfície de 0,016014 $\mathrm{m}^{2}$, utilizados como suporte para as diferentes quantidades de palha estudadas. No interior das unidades experimentais foram posicionados coletores da pulverização abaixo dos fragmentos da palha de aveia-preta, representados por placas de plástico com as mesmas dimensões do fundo das unidades. A recuperação e posterior quantificação da calda de pulverização que transpassou a palha foram efetuadas ao final da aplicação dos tratamentos, após a secagem da pulverização depositada nos respectivos coletores (15 minutos). As palhas foram descartadas e os coletores lavados com $25 \mathrm{~mL}$ de água destilada, dentro de outro recipiente plástico previamente limpo.

As aplicações foram desenvolvidas com auxílio de um equipamento simulador de pulverização, munido de barra posicionada a 0,5 m de altura do solo, equipada com quatro pontas de pulverização espaçadas de 0,5 m, trabalhando em pressão constante e velocidade de $3,6 \mathrm{~km} \mathrm{~h}^{-1}$, para todos os tratamentos estudados. Para simular o herbicida aplicado em pré-emergência, utilizou-se como traçador o corante alimentício Azul Brilhante FDC-1, em solução de 3.000 ppm (calda de pulverização). A determinação da concentração $\left(\mathrm{mg} \mathrm{L}^{-1}\right)$ recuperada do traçador nas soluções obtidas com a lavagem dos coletores foi feita através de curvas-padrão de linearidade, utilizando-se soluções com concentrações conhecidas do corante FDC-1, em água destilada. Essas soluções foram analisadas juntamente com as amostras, na densidade óptica (absorbância) de $630 \mathrm{~nm}$, em espectrofotômetro de UV visível GBC, modelo Cintra 40.
Para melhor compreensão dos resultados, as concentrações originais da solução de lavagem dos coletores, obtidas em unidade de $\mathrm{mg} \mathrm{L}^{-1}$, foram transformadas para porcentagem de transposição, considerando a quantidade coletada no tratamento testemunha $\left(0 \mathrm{t} \mathrm{ha}^{-1}\right)$ igual a $100 \%$ para cada ponta de pulverização.

Os dados de porcentagem de transposição do traçador nas soluções de lavagem foram ajustados ao modelo de Mitscherlich simplificado $\left(\mathrm{Y}=10^{\wedge}\left(2-\left(\mathrm{C}^{*} \mathrm{X}\right)\right)\right)$, de acordo com Maciel (2001), em que: $Y=$ quantidade de traçador que transpôs a palha (\%); C = concavidade da curva em relação a $\mathrm{Y} ; \mathrm{x}=$ quantidade de palha de aveia-preta $\left(\mathrm{t} \mathrm{ha}^{-1}\right)$. Os ajustes foram obtidos com auxílio de um programa estatístico, a partir do qual foram calculados os respectivos coeficientes de determinação, para as diferentes pontas de pulverização estudadas.

\section{RESULTADOS E DISCUSSÃO}

Na Tabela 1 observa-se que foram obtidos elevados valores de coeficientes de determinação $\left(R^{2}\right)$, entre 0,9782 e 0,9971, indicando um ajuste satisfatório dos dados originais de porcentagem de transposição do traçador Azul Brilhante pelo modelo de Mitscherlich simplificado.

Em relação aos diferentes modelos de ponta de pulverização testados, nota-se, de maneira geral, pela Tabela 2 e Figura 1, que houve similaridade na porcentagem de transposição da palha, sendo a diminuição da transposição diretamente proporcional às quantidades de palha. A proximidade entre os valores do parâmetro C (Tabela 1) também indica semelhança entre os resultados, pois se referem às concavidades das curvas, obtidas no ajuste dos dados ao modelo de Mitscherlich simplificado.

As maiores diferenças na porcentagem de transposição da pulverização ocorreram nas quantidades de 1,2 e 4 t de palha ha ${ }^{-1}$. Os modelos das pontas TJ60-11002VS e TXVK-8 proporcionaram as maiores $(51,24,26,25$ e $6,89 \%)$ e menores $(35,38,12,51$ e $1,57 \%)$ porcentagens de transposição direta do traçador nas quantidades de 1, 2 e $4 \mathrm{t} \mathrm{ha}^{-1}$ (Tabela 2), resultando numa diferença percentual entre estas de 15,86, 13,74 e 5,33\%, respectivamente. Esses resultados confirmam uma das 
Tabela 1 - Resultados das análises de regressão das porcentagens de transposição da calda de pulverização, em função dos diferentes modelos de ponta e quantidades de palha de aveia-preta, em relação à condição de ausência de palha, submetidos ao ajuste do modelo de Mitscherlich simplificado

\begin{tabular}{|c|c|c|c|c|c|c|c|}
\hline \multirow{2}{*}{$\begin{array}{l}\text { Análise de } \\
\text { Regressão }\end{array}$} & \multicolumn{7}{|c|}{ Mitscherlich simplificado $\left(\mathrm{Y}=10^{\wedge}(2-(\mathrm{C} * \mathrm{X}))\right)$} \\
\hline & AI1 1002-VS & TT11002-VP & DG11002-VS & FL-5 VS & TJ60-11002 VS & TXVK-8 & XR11002-VS \\
\hline $\mathrm{C}$ & 0,3777 & 0,3737 & 0,3071 & 0,4168 & 0,2904 & 0,4513 & 0,3705 \\
\hline SQ Total & 57269,853 & 57879,991 & 55400,031 & 59264,807 & 56214,744 & 60453,638 & 58323,099 \\
\hline F Regressão & $57173,1 * *$ & $57848,2 * *$ & $55374,3 * *$ & $59230,1^{* *}$ & $56122,8^{* *}$ & $60425,1 * *$ & $58229,1 * *$ \\
\hline $\mathrm{R}^{2}$ & 0,9941 & 0,9971 & 0,9946 & 0,9928 & 0,9883 & 0,9873 & 0,9782 \\
\hline
\end{tabular}

$\mathrm{Y}=$ quantidade de traçador que transpôs a palha $(\%) ; \mathrm{X}=$ quantidade de palha de aveia-preta $\left(\mathrm{t} \mathrm{ha}^{-1}\right) ; \mathrm{C}=$ parâmetro estimado de Mitscherlich simplificado, representado pela concavidade da curva em relação a Y. ** significativo a $1 \%$ de probabilidade.

Tabela 2 - Porcentagem de transposição da calda de pulverização, em função dos diferentes modelos de pontas e quantidades de palha de aveia-preta, em relação à condição de ausência de palha, submetidos ao ajuste do modelo de Mitscherlich simplificado

\begin{tabular}{|c|c|c|c|c|c|c|c|}
\hline \multirow{2}{*}{$\begin{array}{c}\text { Quantidade de } \\
\text { palha } \\
\left.\text { (t ha }^{-1}\right)\end{array}$} & \multicolumn{6}{|c|}{ \% de transposição da pulverização com diferentes modelos de pontas } \\
\cline { 2 - 8 } & AI11002-VS & TT11002-VP & DG11002-VS & FL-5 VS & TJ60-11002 VS & TXVK-8 & XR11002-VS \\
\hline 0 & 100,00 & 100,00 & 100,00 & 100,00 & 100,00 & 100,00 & 100,00 \\
\hline 1 & 41,91 & 42,30 & 49,31 & 38,30 & 51,24 & 35,38 & 42,61 \\
\hline 2 & 17,56 & 17,89 & 24,31 & 14,67 & 26,25 & 12,51 & 18,16 \\
\hline 4 & 3,08 & 3,20 & 5,91 & 2,15 & 6,89 & 1,57 & 3,30 \\
\hline 6 & 0,54 & 0,57 & 1,44 & 0,32 & 1,81 & 0,20 & 0,60 \\
\hline 8 & 0,10 & 0,10 & 0,35 & 0,05 & 0,48 & 0,02 & 0,11 \\
\hline 10 & 0,02 & 0,02 & 0,08 & 0,01 & 0,12 & 0,00 & 0,02 \\
\hline 12 & 0,00 & 0,00 & 0,02 & 0,00 & 0,03 & 0,00 & 0,00 \\
\hline
\end{tabular}

recomendações de uso para o modelo Twinjet (TJ60-11002VS), em função do favorecimento da penetração em resíduos de cultivo pelo liquido pulverizado (Spraying Systems Co., 1999). Além disso, apesar de as pontas TJ6011002 VS e TXVK-8 terem produzido os menores tamanhos de gotas entre todos os outros modelos nas condições estudadas (Christofoletti, 1999a; Spraying Systems Co., 1999), ainda assim tais pontas apresentaram as maiores diferenças entre si nos resultados, indicando a importância do formato dos jatos de pulverização, que, no caso destas, Twinjet (TJ60-11002VS) e Conejet (TXVK-8), são proporcionados pelo jato plano duplo e cônico vazio, respectivamente.

A partir de $6 \mathrm{t}$ de palha ha ${ }^{-1}$, as porcentagens de transposição tornaram-se mais próximas, sendo as diferenças entre a maior e a menor porcentagem de calda pulverizada transposta de 1,$61 ; 0,45 ; 0,12$ e $0,03 \%$, para as quantidades de $6,8,10$ e $12 \mathrm{t}$ de palha ha- ${ }^{-1}$, respectivamente, considerando-se todas as pontas de pulverização estudadas.

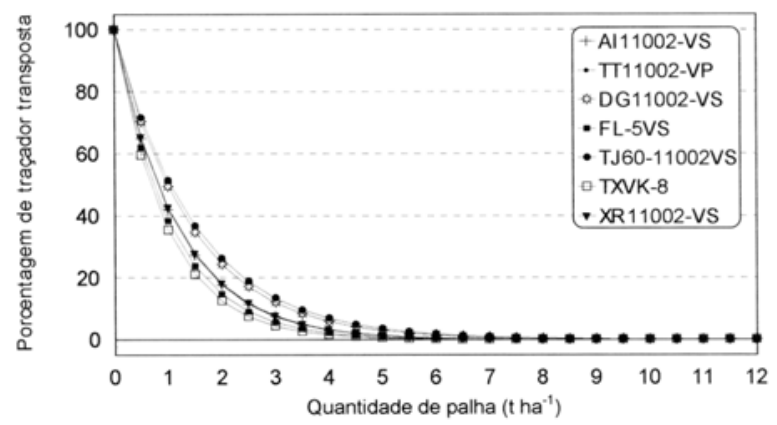

Figura 1 - Efeito do desempenho de pontas de pulverização em porcentagem de transposição da calda de pulverização para diferentes quantidades de palha de aveia-preta, em relação à condição de ausência de palha. Dados ajustados ao modelo de Mitscherlich simplificado. 
Calculando as médias de porcentagem de transposição de todas as pontas de pulverização a partir dos dados apresentados na Tabela 2 , obtêm-se os valores de 43,00; 18,77; 3,73; 0,$78 ; 0,17 ; 0,04$ e 0,01\%, para as quantidades de $1,2,4,6,8,10$ e 12 t de palha ha ${ }^{-1}$, respectivamente. De forma similar, podem ser encontrados elevados valores de retenção de herbicidas em outros ensaios utilizando-se a cobertura morta "aveia-preta" (Avena strigosa), como o de Fornarolli et al. (1998), que constataram $15,0 \%$ da dose do herbicida atrazine transposta pelas quantidades de 4,5 e 9,0 t de palha ha ${ }^{-1}$ ao utilizar a ponta de pulverização TT 11002-VP. Rodrigues et al. (1999) detectaram apenas traços de sulfentrazone no solo em pulverizações com a ponta TP 8002 sobre 6 e $12 \mathrm{t} \mathrm{ha}^{-1}$. Em outro estudo, Rodrigues et al. (2000) observaram menos de 10,0\% de transposição de imazaquin aplicado sobre 7 e $14 \mathrm{t} \mathrm{ha}^{-1}$, utilizando a ponta TP 8002. Maciel (2001) constatou a passagem de 11,3, 1,7 e 0,5\% do traçador Azul Brilhante (FDC-1), aplicado sobre 3, 6 e $9 \mathrm{tha}^{-1}$, respectivamente, ao utilizar a ponta XR 11002-VS.

A partir da quantidade média de 5,9 toneladas por hectare de aveia-preta, produzidas em regiões tradicionais como cobertura morta (Maciel \& Velini, 2002), pode-se considerar que nenhuma das pontas de pulverização estudadas demonstrou desempenho satisfatório para que a calda aplicada transpusesse a palha, pois a média geral foi menor que $1 \%$ do total da transposição, a partir de $6 \mathrm{t}$ de palha ha ${ }^{-1}$. Entretanto, em regiões nas quais as quantidades dessa cobertura morta não ultrapassam $2 \mathrm{t}$ de palha ha ${ }^{-1}$, pode haver um potencial para seleção de modelos das pontas de pulverização mais eficientes, pelo fato de as maiores diferenças médias de transposição encontradas terem sido de 15,86 e 13,74\% para 1 e 2 t de palha $\mathrm{ha}^{-1}$, respectivamente.

Além disso, considerando que a média geral de transposição de calda foi de 43,00\% para $1 \mathrm{t}$ de palha ha $\mathrm{ha}^{-1}$ e $18,77 \%$ para $2 \mathrm{t}$ de palha $\mathrm{ha}^{-1}$, constata-se que quantidades de palha de aveia-preta que podem ser consideradas irrelevantes para condição de sistemas de plantio direto (Almeida, 1988; Maciel, 2001) podem reter boa parte da calda aplicada em operação de pré-emergência. Esse problema pode ser ainda maior para herbicidas que, ao serem inicialmente interceptados e retidos na palha, sejam, posteriormente, pouco retirados pela água da chuva, chegando em baixas quantidades ao solo, como o clomazone (Rodrigues, 1993) e metolachlor (Cavenaghi et al., 2002a).

De maneira geral, a partir dos resultados obtidos na condição do ensaio, pode-se inferir que, em média, todos os modelos de ponta de pulverização mostraram-se similares em termos de transposição da calda pela palha de aveia-preta.

\section{LITERATURA CITADA}

ALMEIDA, F. S. A alelopatia e as plantas. Londrina: IAPAR, 1988. $60 \mathrm{p}$.

CAVENAGHI, A. L. et al. Dinâmica de atrazine e metolachlor aplicados isoladamente e na mistura em tanque sobre a palha de aveia. In: CONGRESSO BRASILEIRO DA CIÊNCIA DAS PLANTAS DANINHAS, 23., 2002, Gramado. Resumos... Londrina: SBCPD, 2002a. p. 161.

CAVENAGHI, A.L. et al. Dinâmica de sulfentrazone em palha de aveia e cana-de-açúcar. In: CONGRESSO BRASILEIRO DA CIÊNCIA DAS PLANTAS DANINHAS, 23., 2002, Gramado. Resumos... Londrina: SBCPD, 2002b. p. 162.

CHRISTOFOLETTI, J. C. Plantio direto e a tecnologia de aplicação de herb icidas. São Paulo: Teejet South America, 1999a. 7 p. (Boletim Técnico, 2).

CHRISTOFOLETTI, J. C. Considerações sobre a deriva nas pulverizações agrícolas e seu controle. São Paulo: Teejet South America, 1999b. 15 p. (Boletim Técnico, 4).

FORNAROLLI, D. A. Influência da cobertura morta no comportamento do herbicida atrazine. 1997. Dissertação (Mestrado em Fitotecnia) - Universidade Estadual de Londrina, Londrina, 1997.

FORNAROLLI, D. A. et al. Influência da cobertura morta no comportamento do herbicida atrazine. Planta Daninha, v. 16, n. 2 , p. $97-107,1998$.

FUNDAÇÃO ABC. Tecnologia de aplicação de defensivo. Castro: Kugler, 1996. 36 p.

MACIEL, C. D. G. Simulação do caminhamento de herbicidas em diferentes tipos e quantidades de palhas utilizadas no sistema de plantio direto. 2001. 89 f. Dissertação (Mestrado em Agronomia/Agricultura) Universidade Estadual Paulista, Botucatu, 2001.

Planta Daninha, Viçosa-MG, v. 22, n. 4, p. 561-566, 2004 
MACIEL, C. D. G.; VELINI, E. D. Levantamento da biomassa seca das coberturas mortas utilizadas no sistema de plantio direto da cultura da soja. In: CONGRESSO BRASILEIRO DA CIÊNCIA DAS PLANTAS

DANINHAS, 23., 2002, Gramado. Resumos.... Londrina: SBCPD, 2002. p. 3.

MARIN, C.D. et al. Washoff of herbicides applied corn residue. Trans ASAE, v. 21, p. 1164-1168, 1978.

PASTANA, F. I. Efeito da retenção de um herbicida pela cobertura morta do solo, no controle de ervas daninhas e na produção do milho com cultivo mínimo. Bragantia, v. 31, n. 22 , p. 259-274, 1972.

RODRIGUES, B. N. Influência da cobertura morta no comportamento dos herbicidas imazaquin e clomazone. Planta Daninha, v. 11, n. 1 e 2, p. 21-28, 1993.

RODRIGUES, B. N. et al. Influência da cobertura morta no comportamento do herbicida trifluralin. Planta Daninha, v. 16, n. 2, p. 163-173, 1998.

RODRIGUES, B. N. et al. Influência da cobertura morta no comportamento do herbicida sulfentrazone. Planta Daninha, v. 17, n. 3, p. 445-458, 1999.
RODRIGUES, B. N. et al. Influência da cobertura morta na retenção do imazaquin em plantio direto de soja. Planta Daninha, v. 18, n. 2, p. 231-239, 2000.

SPRAYING SYSTEMS CO. Teejet - produtos de pulverização para agricultura - catálogo 46-m-br/p.USA: Spraying Systems, 1999. 104 p.

TOFOLI, G. R. et al. Dinâmica de atrazine em palha de aveia e cana-de-açúcar. In: CONGRESSO BRASILEIRO DA CIÊNCIA DAS PLANTAS DANINHAS, 23, 2002,

Gramado. Resumos... Londrina: SBCPD, 2002a. p. 158.

TOFOLI, G. R. et al. Interceptação de diferentes herbicidas e um traçante em palha de aveia, cana-de-açúcar, milheto e pinus. In: CONGRESSO BRASILEIRO DA CIÊNCIA DAS PLANTAS DANINHAS, 23., 2002b, Gramado. Resumos... Londrina: SBCPD, 2002. p. 159.

VELINI, E. D.; NEGRISOLI, E. Controle de plantas daninhas em canacrua. In: CONGRESSO BRASILEIRO DA CIÊNCIA DAS PLANTAS DANINHAS, 22., 2000, Foz do Iguaçu. Palestras... Londrina: SBCPD, 2000. p. 148-164. 